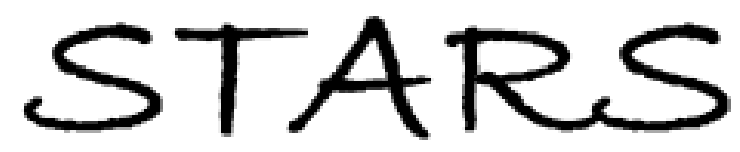

University of Central Florida

STARS

$1-1-2012$

\title{
It's Not Whether You Win or Lose, It's How You Play the Game? The Role of Process and Outcome in Experience Consumption
}

Xiaojing Yang

Huifang Mao

University of Central Florida

Laura A. Peracchio

Find similar works at: https://stars.library.ucf.edu/facultybib2010

University of Central Florida Libraries http://library.ucf.edu

This Article is brought to you for free and open access by the Faculty Bibliography at STARS. It has been accepted for inclusion in Faculty Bibliography 2010 s by an authorized administrator of STARS. For more information, please contactSTARS@ucf.edu.

\section{Recommended Citation}

Yang, Xiaojing; Mao, Huifang; and Peracchio, Laura A., "It's Not Whether You Win or Lose, It's How You Play the Game? The Role of Process and Outcome in Experience Consumption" (2012). Faculty Bibliography 2010s. 3530.

https://stars.library.ucf.edu/facultybib2010/3530

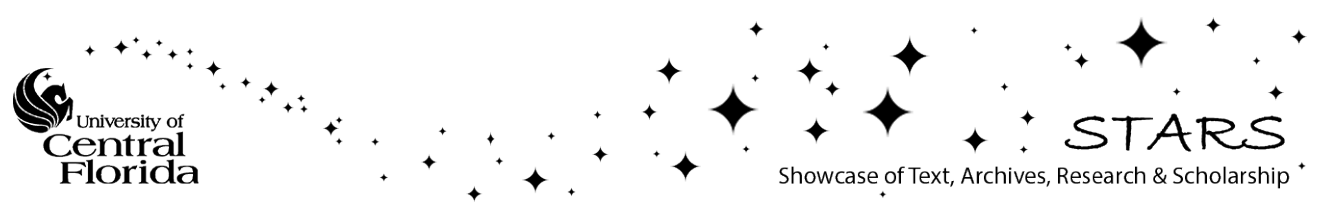


XIAOJING YANG, HUIFANG MAO, and LAURA A. PERACCHIO*

Many firms attempt to enhance experience consumption by facilitating the consumption outcome (i.e., the end state achieved, such as the final score of a basketball game) and the consumption process (i.e., the course through which the end is achieved, such as how the game is played). The authors propose that the roles of outcome and process in the evaluation of experience consumption are dependent not only on consumers' role in the experience (participant vs. spectator) but also on their self-construal (independent vs. interdependent). As a spectator (e.g., watching a game), independents' (vs. interdependents') experience consumption evaluations are more likely to be influenced by outcome, while interdependent (vs. independent) consumers are more likely to be affected by process. The reverse is true when consumers assume the role of a participant in the experience (e.g., playing a game). The authors' theorizing is supported across three studies.

Keywords: experience consumption, process, outcome, self-construal, consumer role

\section{It's Not Whether You Win or Lose, It's How You Play the Game? The Role of Process and Outcome in Experience Consumption}

In the United States, as well as many other parts of the world, experience consumption, such as the pursuit of leisure and entertainment experiences, is increasing (Cave 2010; Etgar 2008; Gilmore and Pine 2007). Many cultural institutions, including the Museum of Modern Art and Art Institute of Chicago, have reported a surge in attendance (Cave 2010), while entertainment at home, such as movie watching, has also increased (Nielsen 2011). According to the Department of Labor's (2011) time use survey, Americans are spending less time shopping and buying products and more time pursuing experiences. Accordingly, Florida (2009) has identified "a tipping point toward something new," with a "shift in the consumption bundle" from "material goods to experiences," labeling this phenomenon as "experience consumption."

\footnotetext{
*Xiaojing Yang is Associate Professor of Marketing, Sheldon B. Lubar School of Business, University of Wisconsin-Milwaukee (e-mail: yangxiao@uwm.edu). Huifang Mao is Associate Professor of Marketing, College of Business Administration, University of Central Florida (e-mail: hmao@bus.ucf.edu). Laura A. Peracchio is Professor of Marketing, Sheldon B. Lubar School of Business, University of WisconsinMilwaukee (e-mail: LPeracch@uwm.edu). The authors thank the JMR review team for their helpful guidance during the review process. Gita Johar served as associate editor for this article.
}

The delivery and marketing of experience consumption differ from traditional product or service marketing in important ways (Etgar 2008; Gilmore and Pine 2007). First, experience consumption includes process as a critical element. Many consumers pursue the consumption of experiences (e.g., watching a basketball game) not only for a particular outcome (e.g., their favorite team wins the game) but also for the process through which the outcome unfolds (e.g., an exciting game). Second, in experience consumption, process and outcome can be inconsistent with, or orthogonal to, each other. For example, a fan may watch a basketball game that is exciting and engaging, but the team the fan supports may lose the game. In such situations, it is important for marketers to know whether consumers' experience consumption evaluations are likely to be relatively favorable because of the enjoyable process or relatively unfavorable because of the unpleasant outcome. In this article, we take a contingency view and explore the conditions that affect the importance of process and outcome on consumers' experience consumption evaluations. Specifically, we propose a theoretical framework that examines a situational factor (i.e., consumers' role in the experience: spectator vs. participant) and an individual difference variable (i.e., self-construal) as moderators of the influence of process and outcome on consumers' experience consumption evaluations. 
The remainder of this article proceeds as follows: We begin by reviewing the literature on experience consumption in marketing, consumer behavior, and psychology. From these related literature streams, we build our framework in terms of the factors that moderate the importance of outcome and process on experience consumption evaluations. Across three studies, we test how the role of consumers in the experience (spectator vs. participant) and their self-construal moderate the effects of these two antecedents.

\section{DETERMINANTS OF THE EVALUATION OF EXPERIENCE CONSUMPTION}

\section{Consumption Outcome and Process}

The importance of outcome in experience consumption is well acknowledged in both marketing and social psychology literature (e.g., Omodei and Wearing 1990; Spreng, MacKenzie, and Olshavsky 1996). Research has long established that outcomes, or the gratifications of end goals, positively affect a person's subjective experience (Deci and Ryan 2000; Trope and Liberman 2003). Much research has found that favorable outcomes enhance experiences by providing closure and meaning to the activity (Csikszentmihalyi 1975; Omodei and Wearing 1990), and consumers are more likely to engage in an experience when desirable outcomes are expected (Liberman and Trope 1998).

Another important driver of experience consumption evaluations-namely, the process of consumption-has received relatively less attention in marketing. Research in social psychology, however, argues that the specific course, or the process through which the end goals are gratified, contributes to positive feelings (Csikszentmihalyi 1975; Trope and Liberman 2003). Positive processes enhance experiences by engaging consumers and promoting absorption in activities such that people often are unaware of the passage of time or distractions from the external environment (Csikszentmihalyi 1975; Novak, Hoffman, and Yung 2000). In contrast, undesirable processes that bring forth pain or discomfort often deter consumers from consumption (Liberman and Trope 1998). In an effort to understand the role of both the end state (outcome) and the course of activities leading to the end state (process), extant literature has found empirical support for both types of antecedents as independent sources of a person's evaluation of experiences (Liberman and Trope 1998; Omodei and Wearing 1990). Accordingly, we adopt the view established in the literature and propose that both outcome and process drive evaluations of experience consumption.

Importantly, construal-level theory and means-end theory (e.g., Trope and Liberman 2003) offer some crucial insights into consumers' mental representations of the process and outcome of an experience. These theories contend that process and outcome are associated with different levels of abstraction (Trope and Liberman 2003; Zhao, Hoeffler, and Zauberman 2007). Specifically, the outcome aspect of an experience is more abstractly construed, while the process is more concretely perceived. Compared with processes, outcomes of an experience (ends) entail more superordinate information, such as desirability, ultimate goals, and purpose (Agrawal and Wan 2009; Trope and Liberman 2003). In contrast, processes, which lead to end states (means), reveal more specific contextualized information regarding how the event unfolds and how the outcome is achieved. Compared with outcomes, processes include subordinate information, such as instrumental goals, feasibility concerns, means, and constraints, and are often mentally represented with greater richness (Agrawal and Wan 2009; Trope and Liberman 2003). In summary, outcomes reflect the superordinate "why" aspect of experience consumption and are relatively more abstract, while processes refer to the subordinate "how" aspects of experience consumption and are relatively more concrete (Trope and Liberman 2003; Vallacher and Wegner 1989).

We contend that both process and outcome contribute to consumers' evaluations of experience consumption and are associated with different levels of abstraction. Our primary goal is to examine the moderating factors that influence the effect of these antecedents. The next section introduces the consumer's role (spectator vs. actor) as one such moderator.

\section{The Moderating Role of Being a Spectator Versus a Participant in an Experience}

Consumers undertake a spectator role in some experience consumption activities (e.g., watching a sports game, listening to a concert) and a participant role in others (e.g., playing sports, singing karaoke) (Pine and Gilmore 1999). We anticipate that consumers focus on process and outcome to varying degrees as a spectator versus a participant when evaluating experience consumption activities. This proposition is rooted in the actor-observer literature, which acknowledges that observers and actors focus on different factors (i.e., abstract, dispositional information vs. concrete, situational information), leading to contrasting views when explaining the same behavior or event (Kelley and Michela 1980; Pronin and Ross 2006).

According to the actor-observer literature, observers have a propensity to explain a behavior by referring to the target person's general and abstract dispositions, traits, or capabilities, while concrete, contextualized, and situational factors are often, to some extent, ignored (Jones and Harris 1967; Jones and Nisbett 1972; Pronin and Ross 2006). Prior research has established this phenomenon, labeled as the "fundamental attribution error" (Ross 1977) or the "correspondence bias" (Gilbert and Malone 1995; Jones and Harris 1967), as robust (Jones 1979; Watson 1982). For example, researchers have shown that the tendency for observers to overly rely on abstract, dispositional information persists even in situations when they are aware of the social constraints imposed on the target person (Gilbert and Malone 1995; Jones 1979). In contrast, actors are more likely to underscore the concrete, situational factors shaping their own behaviors and are less likely to attribute happenings to abstract, dispositional traits (Jones and Harris 1967; Jones and Nisbett 1972; Pronin and Ross 2006).

A substantial amount of empirical evidence offers support for this view (e.g., Nisbett et al. 1973; Pronin and Ross 2006). These studies demonstrate that though people tend to employ abstract, dispositional adjectives (e.g., calm, serious) to judge other people (as observers), when rendering judgments about themselves (as actors), they acknowledge that their behavior is constrained by the specific situation and deem the use of abstract, dispositional adjectives to be 
less appropriate. Moreover, research accounts for the actorobserver differences by the distinct motives actors and observers possess when explaining behaviors and events (e.g., Watson 1982). While observers are motivated to interpret others' behaviors in ways that allow them to better ascertain others' personality traits to predict their behaviors in the future, actors are more compelled to understand their surrounding environment so that they can adapt more effectively.

Extrapolating these findings to a broader consumer cognition context, recent research has documented a construallevel difference between actors and observers (e.g., Trope and Liberman 2010). For example, Fiedler et al. (1995) demonstrate that people in general deploy more concrete linguistic terms to describe themselves while relying more on abstract words to depict others. Likewise, Bar-Anan, Liberman, and Trope (2006), employing the implicit association test, establish that people associate themselves with a concrete level of construal but others with an abstract level of construal. Thus, people are more inclined to rely on concrete information (not only confined to situational factors) when judging their own behaviors (as actors) but abstract information (not only limited to dispositional traits) when assessing others' behaviors (as observers).

In accordance with the previous conclusion that the outcome (process) of experience consumption is relatively more abstract (concrete), we expect that consumers will weigh these antecedents differently when evaluating experience consumption activities. Specifically, spectators (vs. participants), who rely more on abstract information, should be more influenced by the consumption outcome. In contrast, participants (vs. spectators), who are more oriented toward concrete factors, should be affected more by the consumption process. In the next section, we propose a moderating factor (i.e., self-construal) that further influences this relationship.

\section{The Moderating Role of Self-Construal}

Our predictions thus far are based primarily on the actor-observer studies conducted in Western cultures, in which people generally retain a chronic independent selfconstrual. Recent research suggests that the "fundamental attribution error" or the "correspondence bias" does not universally hold across cultures in which people possess different self-construals. Indeed, a considerate amount of evidence shows that people from Eastern interdependent cultures are less likely to be influenced by these effects (e.g., Masuda and Kitayama 2004; Miller 1984; Miyamoto and Kitayama 2002).

In accordance with evidence that actor-observer differences may be culturally specific, we postulate that consumers' self-construal further interacts with their consumer role to influence evaluations of experience consumption. In what follows, we review the literature on self-construal and then provide our rationale for why the type of selfconstrual consumers possess further moderates the effect of outcome and process in experience consumption. Specifically, compared with independent people, who focus more on abstract information as spectators but concrete factors as participants, interdependent spectators (observers) tend to render "actor-like" judgments and are oriented toward concrete information, and interdependent participants (actors) tend to make "observer-like" judgments and focus on abstract information.

Two major types of self-construal-independent and interdependent- have been well established in the literature (Markus and Kitayama 1991). People with an independent self-construal (hereinafter, "independents") accentuate self-related features and minimize the influence of others in the self-schema (Singelis 1994). Conversely, people with an interdependent self-construal (hereinafter, "interdependents") think about themselves more often from a group perspective and value connections and integration with other people (Singelis 1994). A consistent finding in the literature is that interdependents (vs. independents), who value connections and integration with other people and place emphasis on social relationships, are more likely to engage in "perspective taking" (e.g., Wu and Keysar 2007), in which they seek understanding from others' perspectives and respond to others' needs and expectations. Indeed, Markus and Kitayama (1991) contend that people in interdependent cultures are predisposed to read others' minds during interpersonal interactions. The awareness of other people's needs and expectations persists and guides their judgments and behaviors even when these others have not explicitly stated their needs and expectations (e.g., Ambady et al. 1996). Accordingly, because perspective taking enables interdependents to access different informational cues than independents, interdependents should evaluate experience consumption differently.

The spectator role. In contrast with independent spectators, who focus on abstract dispositional information, interdependent spectators, by taking the perspective of the target person, become more aware of the concrete, situational factors that influence that person's behaviors (Church et al. 2003; Markus and Kitayama 1991). Motivated to ascertain the dynamic and intricate characteristics of other people whose needs and expectations vary across contexts, interdependent spectators are more likely to focus on concrete situations when rendering judgments about others and social events, without abstracting traits or features from contexts (Ng and Houston 2006). Empirical studies investigating the prevalence of the "correspondence bias" across independent and interdependent cultures support the position that interdependent observers are likely to make actor-like judgments that incorporate concrete, situational information. From this literature, the tendency of observers to focus on abstract, dispositional information, an effect typically found in Western cultures in which people have a propensity for a chronic independent self-construal, dissipates among Easterners, who tend to have a chronic interdependent selfconstrual (e.g., Masuda and Kitayama 2004; Miyamoto and Kitayama 2002). Instead, Eastern observers are more likely to consider concrete, situational factors when rendering judgments about others.

The participant role. By the same token, when interdependents are actors themselves, they are disposed to engage in perspective taking and render observer-like judgments. Specifically, interdependents are likely to assess their own behaviors from the perspective of an observer. As Cohen and Gunz (2002, p. 55) propose, interdependent actors are likely to "switch their perspective, shifting their attention to experiencing the self from an outsider's perspective or from the perspective of the generalized other." 
Furthermore, they find that interdependent actors are more likely to generate third-person (observer) memories about their own experiences while independent actors tend to have more first-person (actor) memories. Because interdependent actors take the perspective of observers, they assess their own behaviors from a distance and switch the focus from concrete, situational factors to more abstract, dispositional information (Pronin and Ross 2006).

In summary, as a result of perspective taking, interdependents are more attentive to different types of information than independents. When assuming the role of a spectator, compared with independents, who are attentive to abstract, dispositional information, interdependents are more oriented toward concrete, situational factors. In contrast, when assuming a participant role, compared with independents, who focus on concrete, situational factors, interdependents are more predisposed to abstract, dispositional information. As a result, independent (interdependent) spectators' experience consumption evaluations are more likely to be enhanced by favorable experience outcomes (processes), but independent (interdependent) participants' evaluations are likely to be more positive because of favorable experience processes (outcomes).

To examine the moderating factors of the importance of outcome and process on experience evaluations, we focus on the types of experience consumption in which the two evaluation antecedents are inconsistent with each otherthe "positive outcome (but negative process)" experience and the "positive process (but negative outcome)" experience (for similar procedures, see Liberman and Trope 1998; Pham and Avnet 2004; Zhao, Hoeffler, and Zauberman 2007). Examination of these experience types provides clear implications for marketers regarding which components of experiences (process vs. outcome) are crucial for enhancing consumer evaluations. Our theorizing leads us to hypothesize a three-way interaction among self-construal, type of experience (i.e., whether the process or outcome is positive), and consumer role (i.e., participant vs. spectator) on experience evaluations:

$\mathrm{H}_{1}$ : An interaction among self-construal, type of experience, and consumption role will emerge for experience consumption evaluations.

$\mathrm{H}_{1 \mathrm{a}}$ : In the spectator role,

- Independents (vs. interdependents) will have higher evaluations when the outcome is positive (and the process is negative).

- Interdependents (vs. independents) will have higher evaluations when the process is positive (and the outcome is negative).

$\mathrm{H}_{1 \mathrm{~b}}$ : In the participant role,

- Independents (vs. interdependents) will have higher evaluations when the process is positive (and the outcome is negative).

- Interdependents (vs. independents) will have higher evaluations when the outcome is positive (and the process is negative).

\section{STUDY 1}

The primary purpose of Study 1 was to provide evidence for $\mathrm{H}_{1}$. Respondents were asked to recall past experiences in which they assumed the role of either a participant or a spectator. Guidelines for recall were provided so that the experiences featured either a positive outcome (but a negative process) or a positive process (but a negative outcome). We identified respondents' self-construal through measurement scales (Singelis 1994).

In addition to collecting respondents' evaluations of the recalled experiences to test $\mathrm{H}_{1}$, we collected their descriptions and thoughts about the experiences to provide evidence for the mechanism underlying evaluation. Our theorizing posits that the role respondents undertake (participant vs. spectator) and their self-construal will determine the importance they attach to the process and outcome. More specifically, when assuming the role of a spectator, independents (vs. interdependents) should place greater emphasis on the experience outcome, while interdependents (vs. independents) should emphasize the experience process, regardless of whether the experience features a positive outcome or a positive process. In contrast, when undertaking the role of a participant, regardless of the type of the experience, independents (vs. interdependents) should focus more on the process, while interdependents (vs. independents) should emphasize the outcome. Accordingly, we predict a two-way interaction between self-construal and consumer role (spectator vs. participant) on the proportion of outcome- and process-related thoughts respondents generate. More specifically, in the spectator role, independents (vs. interdependents) will generate more thoughts related to the experience outcome but fewer thoughts pertaining to the experience process. In contrast, in the participant role, independents (vs. interdependents) will generate more thoughts with respect to the experience process but fewer thoughts related to experience outcome.

Furthermore, our theorizing predicts that interdependents will be more likely to assume the perspective of others and engage in perspective taking than their independent counterparts (e.g., Wu and Keysar 2007). Interdependents' perspective taking leads them to make "participant-like" judgments when they are spectators by incorporating process information in their evaluations and "spectator-like" judgments when they are participants by concentrating on outcome information. Interdependents (vs. independents) should engage more in perspective taking, regardless of the type of experience or consumer role. Thus, we predict a main effect of self-construal on the proportion of perspective-taking thoughts, such that interdependents will generate a greater proportion of perspective-taking thoughts than independents.

\section{Design and Procedure}

Four hundred thirty-seven respondents participated in a 2 (measured self-construal: independent vs. interdependent) $\times 2$ (type of experience: positive outcome, negative process vs. positive process, negative outcome) $\times 2$ (consumer role: participant vs. spectator) between-subjects full factorial design. All respondents completed a questionnaire at their own pace and were instructed to think about a previous sports consumption experience. We defined sports consumption as a sports match/game between two people or two teams. In the participant condition, respondents were asked to recall a sports game in which they personally played. Examples included playing a basketball game, playing in a tennis match, and playing cards. In the spectator 
condition, respondents were told to recall a game they personally watched. Examples included watching a basketball game, watching a tennis match, and watching a card game.

We manipulated type of experience by varying the guidelines for the recall of the sporting event. In the positive outcome, negative process condition (hereinafter, the "positive outcome" condition), respondents recalled and wrote down a sports consumption experience in which "(1) you [the person/team you support] won the game, and (2) you were satisfied with the match results." At the same time, the event recalled should have a negative process: "(1) the process in the game was frustrating or boring, and (2) you did not enjoy the process." In the negative outcome, positive process condition (hereinafter, the "positive process" condition), respondents recalled and wrote down an experience in which "(1) you [the person/team you support] lost the game, and (2) you were not satisfied with the match results." At the same time, "(1) the process in the game was involving and exciting, and (2) you enjoyed the process." To ensure that our recall instructions did not have any unintended effect on other concepts, two independent coders $(r=.89)$ rated the nature of the recalled experience (ball-related/other; amateur/professional). No differences emerged in the nature of the experience participants recalled across different conditions ( $\mathrm{Fs}<1)$.

After completing the recall task, respondents described in detail the thoughts they had about the sports consumption experience and reported their overall experience consumption evaluations, measured using three sevenpoint scales anchored by "dissatisfied/satisfied," "frustrated/ contented," and "upset/delighted" (Spreng, MacKenzie, and Olshavsky 1996; $\alpha=.88$ ). Next, respondents completed manipulation check measures for both the experience outcome and process. Respondents were asked to ignore the outcome (process) of the game and rate the process (outcome) using the same seven-point scales (outcome: $\alpha=.97$; process: $\alpha=.92$ ). Subsequently, respondents completed two 12-item scales developed by Singelis (1994) that measured their independent and interdependent self-construal. We averaged the items on each scale to form an independence $(\alpha=.81)$ and an interdependence $(\alpha=.81)$ score. According to Singelis (1994), independent and interdependent self-construals reflect different dimensions of the self and can coexist within individuals. Following the procedure outlined in the literature (e.g., Escalas and Bettman 2005; Jain, Desai, and Mao 2007), we included only respondents with a predominant independent or interdependent self in further analyses. Accordingly, we classified respondents as independents (interdependents) whose independence (interdependence) score exceeded the 50th percentile and, at the same time, whose interdependence (independence) score fell below the 50th percentile. With this criterion, 85 independents and 87 interdependents qualified for further analysis. ${ }^{1}$

\footnotetext{
${ }^{1}$ Following the procedure that Escalas and Bettman (2005) outline, we also analyzed the data by employing a continuous index of independence relative to interdependence: (independent score-interdependent score)/(independent score + interdependent score). The regression analysis using the continuous index replicated the findings we report herein.
}

Results

Manipulation checks. As we expected, respondents assigned to the positive outcome (vs. the positive process) condition reported more positive perceptions of the outcome $\left(\mathrm{M}_{\text {positive outcome }}=5.05\right.$ vs. $\mathrm{M}_{\text {positive process }}=4.41$; $\mathrm{F}(1,170)=3.80, p<.05)$ but less positive perceptions of the process $\left(\mathrm{M}_{\text {positive outcome }}=4.75\right.$ vs. $\mathrm{M}_{\text {positive process }}=5.33$; $\mathrm{F}(1,170)=5.92, p<.02)$.

Experience consumption evaluations. We conducted a 2 (self-construal) $\times 2$ (type of experience) $\times 2$ (consumer role) analysis of variance (ANOVA) on overall experience consumption evaluations. As we anticipated, a significant three-way interaction emerged $(\mathrm{F}(1,164)=19.74$, $p<.01$; see Figure 1). For spectators, a significant twoway interaction occurred between self-construal and type of experience $(\mathrm{F}(1,164)=10.88, p<.01)$. Follow-up contrasts revealed that when the outcome was positive, independents (vs. interdependents) reported significantly higher evaluations $\left(\mathrm{M}_{\text {independents }}=5.42\right.$ vs. $\mathrm{M}_{\text {interdependents }}=4.40$; $\mathrm{F}(1,164)=4.65, p<.03)$. In contrast, when the process was positive, interdependents (vs. independents) reported higher evaluations $\left(\mathrm{M}_{\text {independents }}=4.05\right.$ vs. $\mathrm{M}_{\text {interdependents }}=$ 5.19; $\mathrm{F}(1,164)=6.34, p<.01)$. These results suggest that for independent (interdependent) spectators, a positive experience outcome (process) is more likely to enhance evaluations, in support of $\mathrm{H}_{1 \mathrm{a}}$.

For participants, a two-way interaction also occurred between self-construal and type of experience $(F(1,164)=$ $8.89, p<.01)$, but as we anticipated, the pattern was opposite that in the spectator conditions. Planned contrasts showed that when the outcome was positive, interdependents (vs. independents) reported higher evaluations $\left(\mathrm{M}_{\text {independents }}=4.63\right.$ vs. $\mathrm{M}_{\text {interdependents }}=5.55 ; \mathrm{F}(1,164)=$ $4.24, p<.04)$. When the process was positive, independents (vs. interdependents) reported higher evaluations $\left(\mathrm{M}_{\text {independents }}=5.44\right.$ vs. $\mathrm{M}_{\text {interdependents }}=4.47 ; \mathrm{F}(1,164)=$ $4.65, p<.03)$. The contrasts show that a positive experience process (outcome) enhances independent (interdependent) participants' evaluations, in support of $\mathrm{H}_{1 \mathrm{~b}}$.

Experience thoughts. To examine the cognition underlying evaluations, we examined respondents' thoughts about their experience. Two judges who were blind to the treatments classified respondents' thoughts reliably into several categories (interjudge correlation: $\mathrm{r}=.87$; differences were resolved through discussion). First, the judges grouped thoughts according to whether their content was related to the experience process (i.e., how the game unfolded and progressed; e.g., "The game was intense and nailbiting") or outcome (i.e., gains or losses as a result of the game; e.g., "My team lost by a few points"). Second, the judges classified thoughts according to whether they reflected perspective taking (i.e., whether respondents displayed thoughts from the perspectives of other people). Perspective-taking thoughts included "I can relate to the player" and "I can imagine how disappointed the other team must have been." We analyzed outcome-related, processrelated, and perspective-taking thoughts as a proportion of total thoughts. We performed the same three-way ANOVA used previously on these measures.

First, we examined respondents' proportion of outcomeand process-related thoughts. The anticipated two-way interaction (self-construal $\times$ consumer role) was significant 
Figure 1

STUDY 1: EXPERIENCE EVALUATIONS AS A FUNCTION OF SELF-CONSTRUAL, TYPE OF EXPERIENCE, AND CONSUMER ROLE
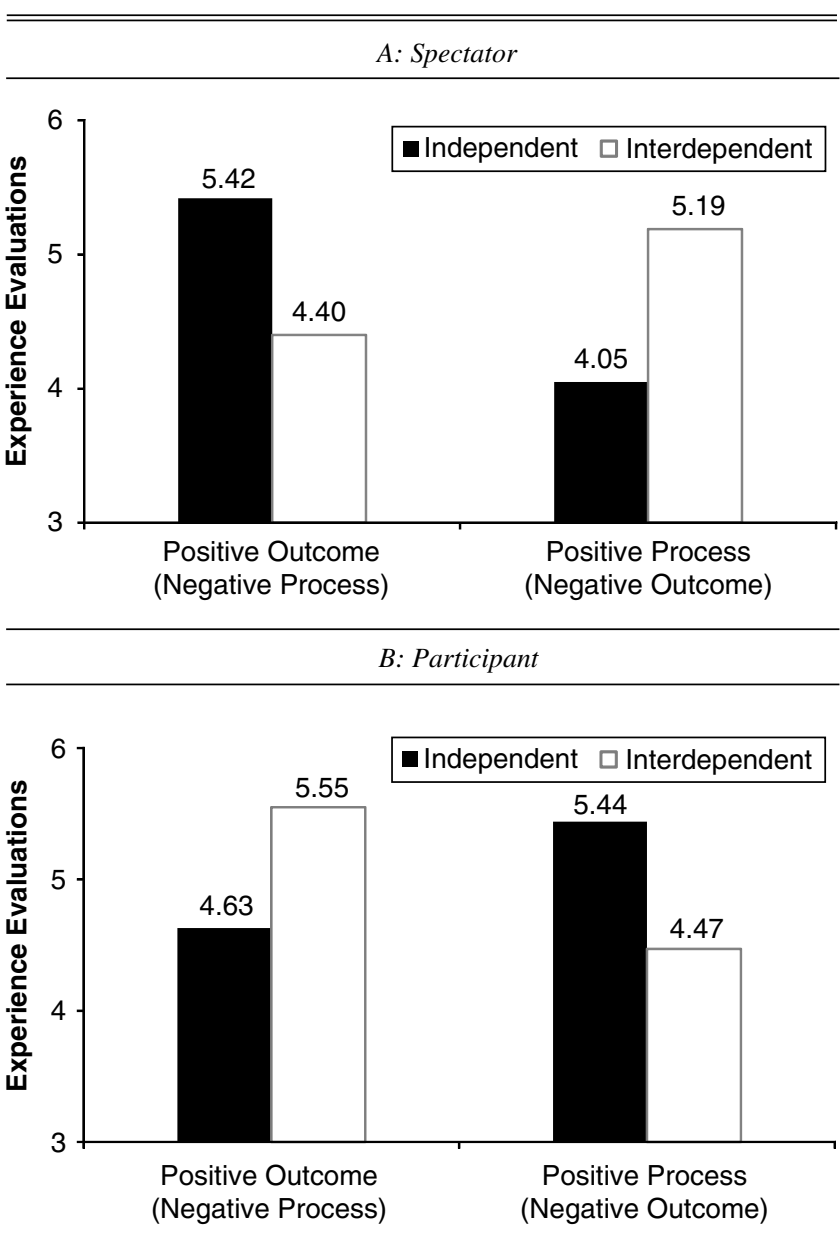

for both outcome-related thoughts $(F(1,164)=20.62$, $p<.01)$ and process-related thoughts $(\mathrm{F}(1,164)=13.77$, $p<.01)$. No other effects emerged (Fs $<1)$. Followup contrasts revealed that when in a spectator role, independents (vs. interdependents) generated a greater proportion of outcome-related thoughts $\left(\mathrm{M}_{\text {independents }}=.38\right.$ vs. $\left.\mathrm{M}_{\text {interdependents }}=.27 ; \mathrm{F}(1,164)=11.30, \quad p<.01\right)$ and a smaller proportion of process-related thoughts $\left(\mathrm{M}_{\text {independents }}=.23\right.$ vs. $\mathrm{M}_{\text {interdependents }}=.37 ; \mathrm{F}(1,164)=8.93$, $p<.01)$. The opposite occurred when respondents assumed the role of a participant. In the participant case, independents (vs. interdependents) generated a smaller proportion of outcome-related thoughts $\left(\mathrm{M}_{\text {independents }}=.28 \mathrm{vs}\right.$. $\left.\mathrm{M}_{\text {interdependents }}=.37 ; \mathrm{F}(1,164)=9.00, p<.01\right)$ and a greater proportion of process-related thoughts $\left(\mathrm{M}_{\text {independents }}=.36 \mathrm{vs}\right.$. $\left.\mathrm{M}_{\text {interdependents }}=.27 ; \mathrm{F}(1,164)=4.77, p<.03\right)$. These results provide further evidence that consumers' focus on either outcome or process in evaluating experience consumption, as determined by both their self-construal and their role in the experience.

Second, we examined respondents' perspective-taking thoughts. As we anticipated, interdependents reported a greater proportion of perspective-taking thoughts than independents $\left(\mathrm{M}_{\text {independents }}=.06\right.$ vs. $\mathrm{M}_{\text {interdependents }}=.21$; $\mathrm{F}(1,164)=11.86, p<.01)$. No other effects were significant for this measure $(\mathrm{Fs}<1)$. Thus, consistent with our theorizing, interdependents (vs. independents) engaged more in perspective taking, regardless of the type of experience or their role in the experience.

Discussion. Using recall of actual consumption experiences and measuring respondents' self-construal, Study 1 revealed a three-way interaction among self-construal, type of experience, and consumer role, providing support for $\mathrm{H}_{1}$. Furthermore, the analysis of thought content offered support for our theorizing. Taken together, these results illustrate the factors that influence the importance of outcome and process in experience consumption evaluations and provide preliminary evidence for the proposed mechanism underlying these evaluations.

\section{STUDY 2}

The major goal of Study 2 was to find further empirical support for our theorizing and bolster the ecological validity of our results by engaging respondents in an actual experience: We asked respondents either to perform in a singing competition (participant condition) or to watch a performance of a singing competition (spectator condition). In addition, Study 2 employed an alternative manipulation of type of experience: After engaging in the singing competition experience, respondents elaborated on the different aspects of the experience to accentuate the salience of either a positive outcome (but a negative process) or a positive process (but a negative outcome). Finally, Study 2 employed a different operationalization of self-construal (i.e., a situational manipulation). Prior research suggests that the self is malleable and thus can be operationalized as an individual difference variable or a situationally activated state (e.g., Ahluwalia 2008).

\section{Design and Procedure}

One hundred eleven respondents participated in a 2 (self-construal: independent vs. interdependent) $\times 2$ (type of experience: positive outcome, negative process vs. positive process, negative outcome) $\times 2$ (consumer role: participant vs. spectator) between-subjects full factorial design. As an overview, after exposure to the self-construal manipulation task, respondents engaged in a singing competition experience either as a participant or a spectator. After that, we manipulated type of experience and measured the dependent variables.

We conducted the experiment individually with each respondent. On arrival, respondents were first exposed to the self-construal manipulation, which was disguised as a purportedly unrelated "proofreading" study (Agrawal and Maheswaran 2005; Kühnen, Hannover, and Schubert 2001). Specifically, respondents were told to read a short paragraph about "a trip to the city" and circle the pronouns in the text. The text was the same across the two conditions with the exception that the 15 pronouns in the text were either independent (I, me, my, and myself) or interdependent (we, us, our, and ourselves). We conducted a pretest to examine whether this manipulation elicited different types of self-construal, as intended. Forty-one respondents were first exposed to the self-construal manipulation and then completed ten statements beginning with "I am 
(ten-statement task; Agrawal and Maheswaran 2005). With Agrawal and Maheswaran's (2005) classification schemes, two independent coders rated the statements as reflecting an independent or an interdependent self-construal (interjudge correlation: $r=.85$; differences were resolved through discussion). Independent statements described a personal attribute, attitude, or ability (e.g., "I am tall"). Interdependent statements described a relationship with others (e.g., "I am a son") or a group membership ("I am a student"). Unrelated statements (e.g., "I am going out for lunch after this") were excluded from analyses. We performed one-way ANOVAs with self-construal as the independent variable on the number of independent and interdependent statements. The results confirmed the success of our manipulation: Respondents in the independent (vs. interdependent) selfconstrual condition generated more self-focused statements $\left(\mathrm{M}_{\text {independents }}=4.23\right.$ vs. $\mathrm{M}_{\text {interdependents }}=2.63 ; \mathrm{F}(1,39)=7.65$, $p<.01)$ and fewer other-focused statements $\left(\mathrm{M}_{\text {independents }}=\right.$ 2.82 vs. $\left.\mathrm{M}_{\text {interdependents }}=4.11 ; \mathrm{F}(1,39)=6.79, p<.01\right)$.

After they completed the self-construal manipulation, respondents were invited to perform as a contestant (participant condition) or watch a contestant performing (spectator condition) in the preliminary round of a singing competition. Respondents were informed that contestants whose performance was favorably judged would proceed to the next round of the competition. In the participant condition, with a microphone attached to a computer, respondents were asked to sing a song of their choice for approximately 30 seconds. Respondents were told that a program installed on the computer would analyze their performance and provide instant feedback afterward. In the spectator condition, respondents were instructed to watch, on a computer, a video taken from a preliminary round of American Idol, in which a contestant sang a song of his or her choice for approximately 30 seconds. To control for the effect of the contestant's gender, we randomly assigned respondents in the spectator condition to watch a performance of either a male or a female contestant. After singing (participant condition) or watching a video of singing (spectator condition), respondents viewed a short video in which the three American Idol judges gave feedback on the singing performance. The feedback video was identical across the participant and spectator conditions and the male and female contestants.

Unlike Study 1, in which respondents evaluated different experiences (experiences with a positive outcome vs. a positive process), we designed Study 2 in such a way that respondents engaged in the same experience, of which the process and outcome entailed both positive and negative aspects (which enabled us to manipulate perceptions of the type of experience, as we describe subsequently). To ensure that the experience outcome was potentially ambiguous, we edited and compiled a feedback video that featured the American Idol judges delivering a mix of positive (e.g., "Great voice. I like everything about you") and negative (e.g., "I was not impressed with your performance") feedback on the singing performance. In addition, we designed the experience process to be potentially ambiguous. On the one hand, we tried to ensure that the instructions for the experience were easy to follow and that respondents could participate in the activity in a quiet and private environment, which contributes favorably to the experience process. On the other hand, the somewhat unorganized setting in the lab and the average quality of the computer's sound system could induce less favorable perceptions of the experience process.

We conducted a pretest to confirm that the experience would indeed elicit mixed perceptions of the outcome and process in both the participant and the spectator conditions. In addition, we included confounding checks to ensure that our manipulation of consumer role did not unintentionally affect other variables. Thirty respondents engaged in the same participant or spectator experience as in the main study and described their perceptions of the experience process and outcome. Next, they completed several confounding checks using seven-point scales: involvement in the experience (e.g., "I felt motivated during the experience"); perceived realism of the experience (e.g., "I found the experience realistic"); and perceived favorability of the outcome, the process, and the overall experience (using the same items as in Study 1). Our analysis showed that across the participant and spectator conditions, respondents generated an equivalent number of positive and negative associations for both process and outcome (Fs $<1$ ), confirming that the experience indeed induced positive and negative perceptions. In addition, ANOVAs performed on the potential confounding variables revealed that the spectator and participant conditions evoked a comparable level of involvement $\left(\mathrm{M}_{\text {participant }}=5.31\right.$ vs. $\left.\mathrm{M}_{\text {spectator }}=5.03\right)$, realism $\left(\mathrm{M}_{\text {participant }}=4.46\right.$ vs. $\left.\mathrm{M}_{\text {spectator }}=4.44\right)$, favorability of the process $\left(\mathrm{M}_{\text {participant }}=5.03\right.$ vs. $\left.\mathrm{M}_{\text {spectator }}=5.16\right)$, outcome $\left(\mathrm{M}_{\text {participant }}=5.19\right.$ vs. $\left.\mathrm{M}_{\text {spectator }}=5.09\right)$, and overall experience $\left(M_{\text {participant }}=4.89\right.$ vs. $\left.M_{\text {spectator }}=5.22\right)($ all Fs $<1)$.

After the singing competition experience, respondents completed a booklet at their own pace, which consisted of the manipulation of type of experience, followed by an assessment of respondents' evaluations of the experience. To manipulate type of experience, we instructed respondents to describe the singing competition experience in which they had taken part. Guidelines for the descriptions varied to accentuate the positive outcome (and negative process) or the positive process (and negative outcome) of the experience. In the positive outcome condition, respondents were asked to describe both negative aspects of the process (e.g., the somewhat unorganized setting) and positive aspects of the outcome (e.g., likely to qualify for next round). In the positive process condition, respondents were instructed to elaborate on both positive aspects of the process (e.g., a private environment) and negative aspects of the outcome (e.g., may not proceed to the next round).

Using the same checks as in Study 1 (outcome: $\alpha=.78$; process: $\alpha=.75)$, a pretest $(n=60)$ confirmed that our manipulation of type of experience was successful. Specifically, respondents in the positive outcome (vs. positive process) condition reported more positive perceptions of the outcome $\left(\mathrm{M}_{\text {positive outcome }}=5.20\right.$ vs. $\mathrm{M}_{\text {positive process }}=4.61$; $\mathrm{F}(1,56)=6.22, p<.02)$ but less positive perceptions of the process $\left(M_{\text {positive outcome }}=4.78\right.$ vs. $M_{\text {positive process }}=5.25$; $\mathrm{F}(1,56)=4.09, p<.05)$. Moreover, to provide a manipulation check for consumer role, we asked respondents to indicate their level of agreement on a four-item scale (e.g., "I am a spectator of the game," "I am a participant of the game (reverse coded)"; 1 = "strongly disagree," and 7 = "strongly agree"; $\alpha=.88$ ). We averaged the items to form an index of consumer role, with higher (lower) scores indicating a spectator (participant) role. Respondents in the spectator 
(vs. participant) condition confirmed their role as a spectator $\left(\mathrm{M}_{\text {participant }}=2.13\right.$ vs. $\mathrm{M}_{\text {spectator }}=5.98 ; \mathrm{F}(1,56)=252.27$, $p<.001)$.

After completing the manipulation of type of experience, respondents provided their overall evaluations of the experience using the same scale employed in Study $1(\alpha=.77)$. Finally, respondents were thanked and debriefed.

\section{Results}

Experience consumption evaluations. We performed a 2 (self-construal) $\times 2$ (type of experience $) \times 2$ (consumer role) ANOVA on respondents' experience consumption evaluations. Consistent with the findings in Study 1, a significant three-way interaction emerged $(\mathrm{F}(1,103)=$ $18.63, p<.01$; see Figure 2). For spectators, a significant two-way interaction occurred between self-construal and type of experience $(\mathrm{F}(1,103)=8.32, p<.01)$. Followup contrasts revealed that when the positive outcome was made salient, independents (vs. interdependents) reported significantly higher evaluations of the singing competition experience $\left(\mathrm{M}_{\text {independents }}=4.69\right.$ vs. $\mathrm{M}_{\text {interdependents }}=4.00$; $\mathrm{F}(1,103)=4.18, p<.04)$. In contrast, when the positive process was salient, interdependents (vs. independents) reported higher evaluations $\left(\mathrm{M}_{\text {independents }}=4.49\right.$ vs. $\left.\mathrm{M}_{\text {interdependents }}=5.22 ; \mathrm{F}(1,103)=4.05, p<.04\right)$. For participants, a significant two-way interaction also occurred between self-construal and type of experience $(\mathrm{F}(1,103)=$ $10.30, p<.01)$. Planned contrasts revealed that when the positive outcome was salient, interdependents (vs. independents) reported higher evaluations of the experience $\left(\mathrm{M}_{\text {independents }}=4.18\right.$ vs. $\mathrm{M}_{\text {interdependents }}=5.05 ; \mathrm{F}(1,103)=$ $5.46, p<.02)$. However, when the positive process was salient, independents (vs. interdependents) reported higher evaluations $\left(\mathrm{M}_{\text {independents }}=5.25\right.$ vs. $\mathrm{M}_{\text {interdependents }}=4.41$; $\mathrm{F}(1,103)=4.86, p<.03)$.

Discussion. The findings of Study 2 augment the robustness of our theorizing by engaging respondents in an actual experience (a singing competition) and by employing an alternative operationalization of self-construal and type of experience. Converging evidence for our theorizing emerged across both Studies 1 and 2.

Study 3 addresses several important questions. Our theorizing contends that perspective taking is a crucial psychological process that accounts for why interdependents differ from independents in experience consumption evaluations. Although the thought-content analysis in Study 1 provided preliminary support for the perspective-taking account, Study 3 seeks additional evidence for this psychological mechanism. Specifically, Study 3 examines whether inhibiting perspective-taking tendency-that is, encouraging people to keep their own perspectives rather than considering someone else's-leads interdependents to evaluate experience consumption in the same manner as independents. According to our theorizing, when perspective taking is suppressed, regardless of consumers' self-construal, spectators (vs. participants) should favor an experience with a positive outcome, while participants (vs. spectators) should evaluate an experience with a positive process more favorably.

Furthermore, in Study 2 we manipulated outcome and process by altering respondents' perceptions. To extend the robustness of our findings, Study 3 manipulated actual experience. Thus, respondents engaged in experiences that differed in terms of outcome and process.
Figure 2

STUDY 2: EXPERIENCE EVALUTIONS AS A FUNCTION OF SELF-CONSTRUAL, TYPE OF EXPERIENCE, AND CONSUMER ROLE

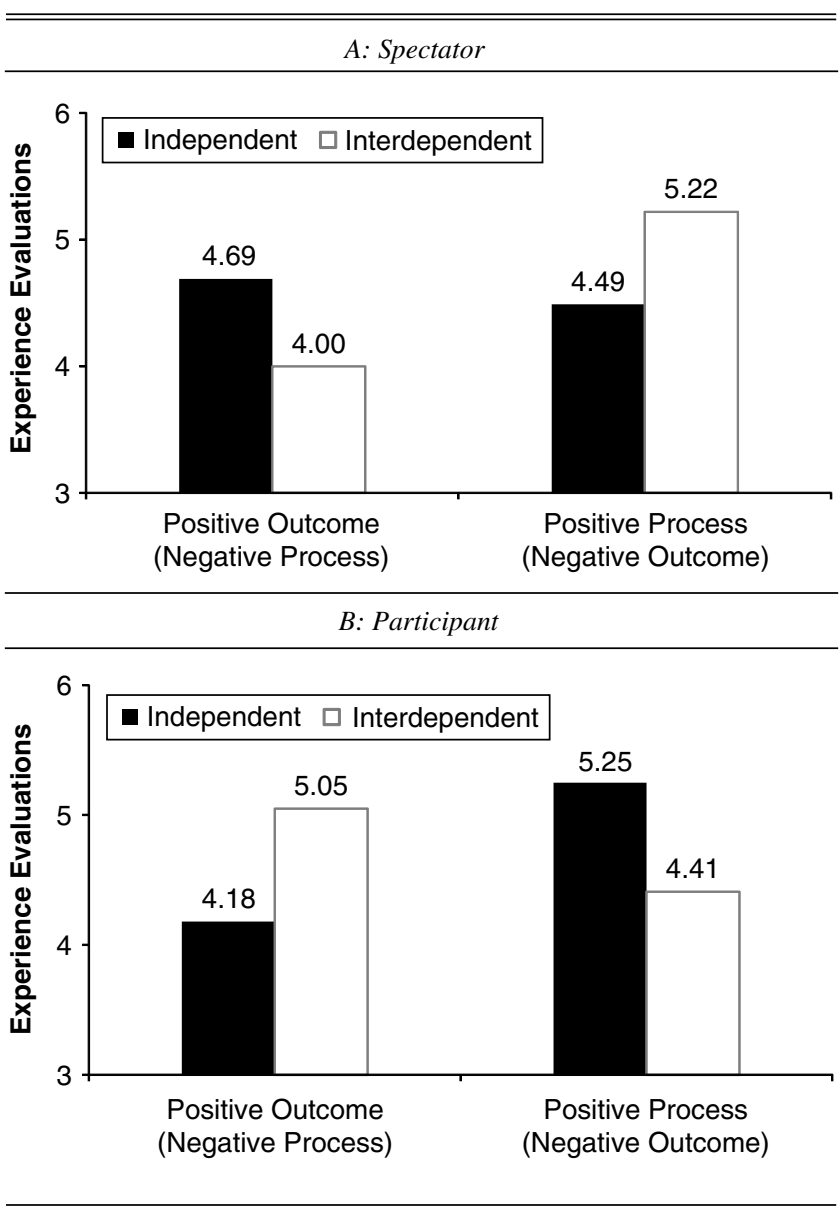

STUDY 3

Study 3 manipulated the key psychological mechanism, perspective taking. In the control condition, in which perspective taking is not suppressed and respondents act on their own propensity to take the perspective of another (similar to Studies 1 and 2), respondents' experience evaluations should replicate our previous results. That is, independent (vs. interdependent) spectators should generate more favorable evaluations of an experience with a positive outcome (vs. process), and independent (vs. interdependent) participants should offer more favorable evaluations of an experience with a positive process (vs. outcome). Conversely, in conditions in which perspective taking is suppressed, by priming respondents to maintain their own perspectives, interdependents should evaluate the experiences in the same way as independents. Thus:

$\mathrm{H}_{2}$ : An interaction among self-construal, type of experience, consumption role, and perspective taking will emerge for experience consumption evaluations.

$\mathrm{H}_{2 \mathrm{a}}$ : In the absence of perspective-taking suppression primes (the control condition), the same interaction among selfconstrual, type of experience, and consumer role as in Studies 1 and 2 will be replicated. 
$\mathrm{H}_{2 \mathrm{~b}}$ : When respondents' perspective-taking tendency is suppressed, an interaction between consumer role and type of experience will occur. Specifically,

- In an experience with a positive outcome (and a negative process), spectators (vs. participants) will have higher evaluations.

- In an experience with a positive process (and a negative outcome), participants (vs. spectators) will have higher evaluations.

\section{Design and Procedure}

Two hundred thirty respondents participated in a 2 (selfconstrual: independent vs. interdependent) $\times 2$ (type of experience: positive outcome, negative process vs. positive process, negative outcome) $\times 2$ (consumer role: participant vs. spectator) $\times 2$ (perspective taking: suppressed vs. control) between-subjects full factorial design. On arrival, respondents were first exposed to the same self-construal manipulation as in Study 2. Next, to manipulate perspective taking, we asked respondents to recall and describe two consumption experiences. To suppress perspective taking, we asked half the respondents to recall and briefly describe two experiences in which they stuck with their own perspectives (e.g., making a decision without considering other people's perspectives). In the control condition, the other half recalled two experiences that happened in the past. A pretest $(n=33)$ confirmed that our manipulation of perspective taking was successful, using two sevenpoint scales (e.g., "I tried to look from others' perspective"; $\mathrm{r}=.52, p<.01)$. Respondents in the perspective-taking suppression condition were less likely to take the perspectives of others $\left(\mathrm{M}_{\text {suppression }}=3.00\right.$ vs. $\mathrm{M}_{\text {control }}=4.67 ; \mathrm{F}(1,31)=$ $4.93, p<.03)$.

Next, respondents took part in a singing competition experience similar to Study 2, in which they either performed as a contestant in a singing competition or watched a video of a contestant performing. In this study, the actual outcome and process of the singing competition experience were manipulated. Respondents in the positive outcome condition took part in a singing competition experience in which the process was negative and the outcome was favorable. To manipulate the unfavorable process, respondents heard a preprogrammed beep every five seconds while singing (or watching the contestant). In addition, the lab room appeared in a disorganized and untidy state, with unfinished food and scraps of paper scattered across the room. To highlight the positive outcome, after they finished singing (or watching the video), respondents watched a feedback video that featured the American Idol judges delivering positive comments about them (or the contestant they watched) (e.g., "Great voice. I like everything about you"). They were informed that they themselves (or the contestant they watched) qualified for the next round of the singing competition.

In contrast, respondents in the positive process condition took part in a singing competition experience, which featured a favorable process but a negative outcome. To manipulate the positive process, respondents sang (or watched the contestant sing) in a quiet environment, without beeping. In addition, the lab room appeared in an organized and tidy state, with no unfinished food or scraps of paper. To highlight the negative outcome, after they finished singing (or watching the video), respondents watched a feedback video that featured the American Idol judges delivering negative comments about them (or the contestant they watched) (e.g., "I was not impressed with your performance"), and they were (or the contestant they watched was) eliminated and disqualified for the next round of the singing competition.

We conducted a pretest (2 [type of experience: positive outcome vs. positive process] $\times 2$ [consumer role: spectator vs. participant]) to provide evidence for our manipulations and to ensure that our manipulations did not unintentionally affect other variables. Eighty-three respondents engaged in the same experience as in the main study and completed the manipulation check measures of type of experience and consumer role, using the same scales as in Study 2 (outcome: $\alpha=.95$; process: $\alpha=.91$; consumer role: $\alpha=.92$ ). In addition to the confounding variables examined in Study 2 (involvement, realism of the experience), we measured respondents' mood using the PANAS (Positive Affect, Negative Affect Schedule; Waston, Clark, and Tellegen 1988). The ANOVAs performed on confounding checks showed that our manipulations did not inadvertently affect the potential confounding variables (all Fs $<1$ ). The results on manipulation checks also revealed support for our manipulations. Specifically, respondents in the positive outcome (vs. positive process) condition reported more positive perceptions of the outcome $\left(\mathrm{M}_{\text {positive outcome }}=5.13 \mathrm{vs}\right.$. $\left.\mathrm{M}_{\text {positive process }}=4.38 ; \mathrm{F}(1,79)=4.71, p<.03\right)$ but less positive perceptions of the process $\left(\mathrm{M}_{\text {positive outcome }}=4.51 \mathrm{vs}\right.$. $\left.\mathrm{M}_{\text {positive process }}=5.21 ; \mathrm{F}(1,79)=6.80, p<.01\right)$. In addition, those in the spectator (vs. participant) condition confirmed their role as a spectator $\left(\mathrm{M}_{\text {participant }}=2.15\right.$ vs. $\mathrm{M}_{\text {spectator }}=$ $6.36 ; \mathrm{F}(1,79)=279.83, p<.001$; higher scores indicated a spectator role).

After the singing competition experience, respondents completed a booklet at their own pace and evaluated the singing competition experience using the same scales employed in the previous studies $(\alpha=.90)$. Respondents assigned to the participation condition also indicated whether they indeed sang. ${ }^{2}$ Finally, respondents were thanked and debriefed.

\section{Results}

Experience consumption evaluations. We conducted a 2 (self-construal) $\times 2$ (type of experience) $\times 2$ (consumer role) $\times 2$ (perspective taking: suppressed vs. control) ANOVA on respondents' experience consumption evaluations. We anticipate that in the control condition, in which perspective taking is not suppressed, the results will replicate the previous findings. Conversely, when perspective taking is suppressed, interdependents should evaluate the experience in the same way as the independents. As we expected, a significant four-way interaction of the independent variables emerged $(\mathrm{F}(1,204)=10.17, p<.01$; see Figure 3).

We conducted follow-up analyses to understand this interaction. As we hypothesized, in the control condition, the findings in Studies 1 and 2 were replicated, and the same three-way interaction among self-construal, type

\footnotetext{
${ }^{2}$ Ten respondents reported that they did not sing during the experiment, so we excluded their data from the analysis (including these data did not change the results).
} 
Figure 3

STUDY 3: EXPERIENCE EVALUTIONS AS A FUNCTION OF SELF-CONSTRUAL, TYPE OF EXPERIENCE, CONSUMER ROLE, AND PERSPECTIVE TAKING

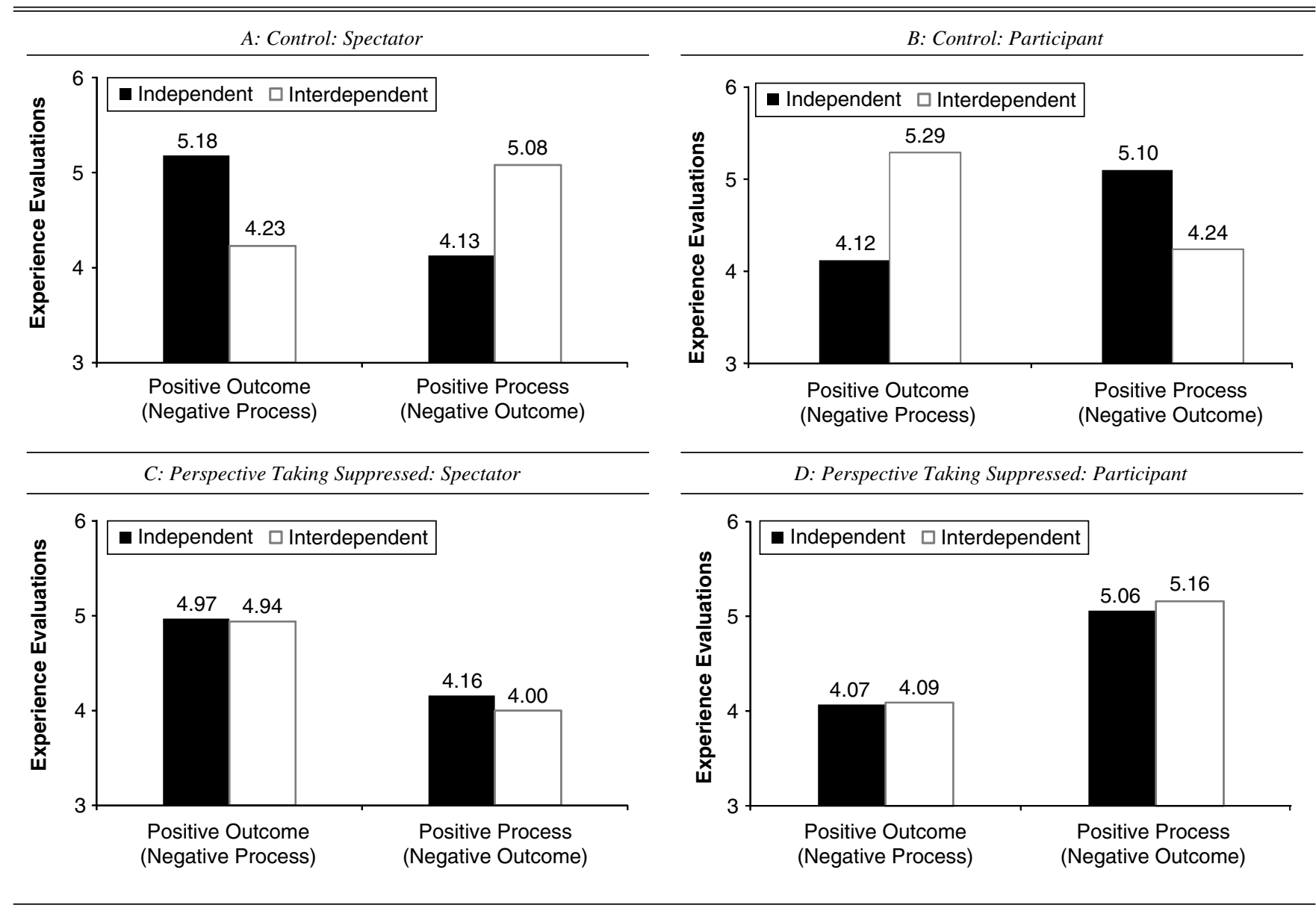

of experience, and consumer role emerged $(\mathrm{F}(1,204)=$ $19.37, p<.01)$. For spectators, a significant two-way interaction between self-construal and type of experience emerged $(\mathrm{F}(1,204)=9.57, p<.01)$. Follow-up contrasts revealed that when the experience featured a positive outcome, independents (vs. interdependents) reported significantly higher evaluations $\left(\mathrm{M}_{\text {independents }}=5.18\right.$ vs. $\left.\mathrm{M}_{\text {interdependents }}=4.23 ; \mathrm{F}(1,204)=4.42, p<.04\right)$. In contrast, when the experience had a positive process, interdependents (vs. independents) reported higher evaluations $\left(\mathrm{M}_{\text {independents }}=4.13\right.$ vs. $\mathrm{M}_{\text {interdependents }}=5.08 ; \mathrm{F}(1,204)=$ $5.20, p<.02)$. For participants, a significant two-way interaction between self-construal and type of experience also occurred $(\mathrm{F}(1,204)=9.46, p<.01)$. Planned contrasts revealed that when the experience featured a positive outcome, interdependents (vs. independents) reported higher evaluations $\left(\mathrm{M}_{\text {independents }}=4.12\right.$ vs. $\mathrm{M}_{\text {interdependents }}=5.29$; $\mathrm{F}(1,204)=5.91, p<.02)$. However, when the experience had a positive process, independents (vs. interdependents) reported marginally higher evaluations $\left(\mathrm{M}_{\text {independents }}=5.10\right.$ vs. $\left.\mathrm{M}_{\text {interdependents }}=4.24 ; \mathrm{F}(1,204)=3.63, p<.06\right)$. Thus, the results support $\mathrm{H}_{2 \mathrm{a}}$.

In contrast, when respondents' perspective-taking tendency was suppressed, a two-way interaction between consumer role and type of experience emerged $(F(1,204)=$
$16.70, p<.01)$. The results revealed that interdependents and independents evaluated the experience in the same way. Planned contrasts showed that in the positive outcome condition, spectators reported more favorable evaluations of the experience than participants $\left(\mathrm{M}_{\text {spectators }}=4.96 \mathrm{vs}\right.$. $\left.\mathrm{M}_{\text {participants }}=4.08 ; \mathrm{F}(1,204)=6.45, p<.02\right)$. The opposite occurred in the positive process condition, such that participants evaluated the experience more positively than spectators $\left(\mathrm{M}_{\text {spectators }}=4.08\right.$ vs. $\mathrm{M}_{\text {participants }}=5.12 ; \mathrm{F}(1,204)=$ $10.76, p<.01)$. Thus, the results also support $\mathrm{H}_{2 \mathrm{~b}}$.

Discussion. Study 3 offers strong empirical support for the mechanism posited to underlie the evaluation effects: perspective taking. Importantly, when perspective taking was suppressed, interdependents evaluated the experience in the same way as independents. In addition, in the control condition, we provide converging evidence for the threeway interaction among self-construal, type of experience, and consumer role by varying the outcome and process of an actual experience.

\section{GENERAL DISCUSSION}

\section{Findings and Contributions}

The empirical results across three studies reveal that when people evaluate experience consumption, the impor- 
tance of process and outcome depends on two factors: consumers' role in the experience and self-construal. When assuming the role of a spectator, independents (vs. interdependents) rely more on outcome while interdependents (vs. independents) focus on process in experience consumption evaluations. Conversely, when undertaking the role of a participant, independents (vs. interdependents) focus more on process while interdependents (vs. independents) rely more on outcome.

This research contributes to the marketing literature in various ways. First, this work delineates the conditions under which the process, along with the outcome, plays a significant role in determining experience consumption evaluations. Second, this research elucidates the underlying mechanism for the identified effects. Independents (vs. interdependents) focus more on abstract information when they are spectators and evaluate experiences more on the consumption outcome, while interdependent spectators, who take the perspective of participants, base their evaluation more on the consumption process. Conversely, independent (vs. interdependent) participants rely more on process in their evaluations, while interdependent (vs. independent) participants, taking the perspective of spectators, rely more on outcome.

Finally, this research extends the actor-observer literature. The results suggest that self-construal moderates the actor-observer paradigm. We find that independents behave consistently with the actor-observer paradigm and consider the concrete, contextual information of experience consumption when they are actors but not observers. However, the findings for interdependents reveal a different pattern. Interdependents tune in to others' opinions and, as a result, make relatively more actor-like judgments when they are observers and more observer-like judgments when they are actors. That is, interdependents (vs. independents) are more (less) likely to consider concrete, contextual information when they are observers (actors).

\section{Managerial Implications}

An increasing number of consumers are pursuing experiential activities (e.g., using social networks, practicing yoga at a gym, going to theme parks). Many experience consumption activities contain both process and outcome components. For example, in social networking, the process aspect might include whether the website provides tools for consumers to interact with their friends (e.g., forums, pictorial editing tools, templates), whether surfing on the website is smooth and effortless, and so forth; the outcome aspect might entail things such as whether consumers are able to keep connected with their friends and make new friends.

Our research has significant, practical implications because it offers insights into how managers can optimally allocate resources to areas that maximally enhance experience consumption evaluations. Our findings suggest that depending on the consumer segments targeted (i.e., independents or interdependents) and the type of consumer experience offered (i.e., whether consumers assume the role of spectators or participants in the experience), companies can reap greater benefits by focusing on enhancing the process or the outcome of the experience. Extant research suggests that marketers can employ self-construal as an effective way to segment consumers (e.g., Ahluwalia 2008).
For example, consumers in North America (vs. East Asia), male (vs. female) consumers, and European American (vs. Asian and Hispanic American) consumers are likely to possess an independent (vs. interdependent) self-construal (e.g., Ahluwalia 2008; Cross, Morris, and Gore 2002). Our research suggests that marketing efforts targeting independent (interdependent) consumers will be more effective if marketers allocate resources to the process (outcome) of the experience when these consumers are participants; in contrast, if consumers are spectators, efforts enhancing the outcome (process) will be more effective for independent (interdependent) consumers.

In addition to enhancing the process (e.g., providing necessary tools for consumers to achieve a positive outcome, making a game more entertaining) and the outcome (e.g., gratifying consumers' desires and expectations) directly, Study 2 sheds light on how marketers can influence consumers' evaluations of experience consumption activities, for which consumers' perceptions of the process or outcome may be ambiguous. We show that making certain aspects of the experience salient in consumers' minds can lead them to perceive and evaluate the experience differently. Therefore, in addition to allocating resources to improve the process versus outcome, marketers could enhance evaluations by encouraging consumers to focus on positive aspects of the outcome or process, whichever is more relevant to the overall experience.

\section{Future Research Directions}

Further research should investigate factors that might have an impact on our results. For example, Ahluwalia (2008) shows that information-processing differences between interdependents and independents are more prominent when consumers engage in extensive elaboration. Thus, research could examine whether our results, obtained in a setting in which respondents likely had moderately high motivation, are mitigated when motivation is low. In addition, we focus on experience consumption evaluations, and thus further research could examine whether our results generalize to more traditional types of consumption (e.g., toothpaste). Another point that warrants further investigation is whether perceptions of outcome and process affect each other for some experience consumption activities. For example, consumers may find the experience process more enjoyable when the outcome is desirable. In this work, as we manipulated process and outcome, respondents' perceptions of these two experience components were less likely to influence each other. Thus, further research could examine consumption situations in which perceptions of process and outcome might be intertwined.

\section{REFERENCES}

Agrawal, Nidhi and Durairaj Maheswaran (2005), "The Effects of Self-Construal and Commitment on Persuasion," Journal of Consumer Research, 31 (4), 841-49.

_ and Echo Wen Wan (2009), "Regulating Risk or Risking Regulation? Construal Levels and Depletion Effects in the Processing of Health Messages," Journal of Consumer Research, 36 (3), 448-62.

Ahluwalia, Rohini (2008), "How Far Can a Brand Stretch? Understanding the Role of Self-Construal," Journal of Marketing Research, 45 (June), 337-50. 
Ambady, Nalini, Jasook Koo, Fiona Lee, and Robert Rosenthal (1996), "More Than Words: Linguistic and Nonlinguistic Politeness in Two Cultures," Journal of Personality and Social Psychology, 70 (5), 996-1011.

Bar-Anan, Yoav, Nira Liberman, and Yaacov Trope (2006), "The Association Between Psychological Distance and Construal Level: Evidence from an Implicit Association Test," Journal of Experimental Psychology: General, 135 (4), 609-622.

Cave, Damien (2010), "In Recession, Americans Doing More, Buying Less," The New York Times, (January 2), [available at http://www.nytimes.com/2010/01/03/business/economy/ 03experience.html?pagewanted=all].

Church, Timothy A., Fernando A. Ortiz, Marcia S. Katigbak, Tatyana V. Avdeyeva, Alice M. Emerson, José de Jesús Vargas Flores, and Joselina Ibáñez Reyes (2003), "Measuring Individual and Cultural Differences in Implicit Trait Theories," Journal of Personality and Social Psychology, 85 (2), 332-47.

Cohen, Dov and Alex Gunz (2002), "As Seen by the Other...: Perspectives on the Self in the Memories and Emotional Perceptions of Easterners and Westerners,"Psychological Science, 13 (1), 55-59.

Cross, Susan E., Michael L. Morris, and Jonathan S. Gore (2002), "Thinking About Oneself and Others: The RelationalInterdependent Self-Construal and Social Cognition," Journal of Personality and Social Psychology, 82 (3), 399-418.

Csikszentmihalyi, Mihaly (1975), Beyond Boredom and Anxiety. San Francisco: Jossey-Bass.

Deci, Edward L. and Richard Ryan (2000), "The 'What' and 'Why' of Goal Pursuits: Human Needs and the SelfDetermination of Behavior,' Psychological Inquiry, 11 (4), 227-68.

Department of Labor (2011), "American Time Use Survey (ATUS)," Bureau of Labor Statistics, United States Department of Labor [available at http://www.bls.gov/news.release/atus .nr0.htm].

Escalas, Jennifer E. and James R. Bettman (2005), "SelfConstrual, Reference Groups, and Brand Meaning," Journal of Consumer Research, 32 (December), 378-89.

Etgar, Michael (2008), "A Descriptive Model of the Consumer Co-Production Process," Journal of the Academy of Marketing Science, 36 (1), 97-108.

Fiedler, Klaus, Guin R. Semin, Catrin Finkenauer, and Ingnd Berkel (1995), "Actor-Observer Bias in Close Relationships: The Role of Self-Knowledge and Self-Related Language," Personality and Social Psychology Bulletin, 21 (5), 525-38.

Florida, Richard (2009), "The New Normal?" (May 6), (accessed July 12, 2012), [available at http://www.creativeclass.com/ creative_class/2009/05/06/the-new-normal].

Gilbert, Daniel T. and Patrick S. Malone (1995), "The Correspondence Bias," Psychological Bulletin, 117 (1), 21-38.

Gilmore, James H. and B. Joseph Pine (2007), Authenticity: What Consumers Really Want. Boston: Harvard Business School Press.

Jain, Shailendra Pratap, Kalpesh Kaushik Desai, and Huifang Mao (2007), "The Influence of Chronic and Situational SelfConstrual on Categorization," Journal of Consumer Research, 34 (1), 66-76.

Jones, Edward E. (1979), “The Rocky Road from Acts to Dispositions," American Psychologist, 34 (2), 107-117.

and Victor A. Harris (1967), "The Attribution of Attitudes,"Journal of Experimental Social Psychology, 3 (1), 1-24. and Richard E. Nisbett (1972), "The Actor and the Observer: Divergent Perceptions of the Cause of Behavior," in Attribution: Perceiving the Causes of Behavior, Edward E. Jones, David E. Kanouse, Harold H. Kelley, Richard E. Nisbett, Stuart S. Valins, and Bernard Weiner, eds. Morristown, NJ: General Learning, 79-94.

Kelley, Harold H. and John L. Michela (1980), "Attribution Theory and Research," Annual Review of Psychology, 31, 457-501.

Kühnen, Ulrich, Bettina Hannover, and Benjamin Schubert (2001), "The Semantic-Procedural Interface Model of the Self," Journal of Personality and Social Psychology, 80 (3), 397-409.

Liberman, Nira and Yaacov Trope (1998), "The Role of Feasibility and Desirability Considerations in Near and Distant Future Decisions: A Test of Temporal Construal Theory," Journal of Personality and Social Psychology, 75 (July), 5-18.

Markus, Hazel R. and Shinobu Kitayama (1991), "Culture and the Self: Implications for Cognition, Emotion, and Motivation," Psychological Review, 98 (2), 224-53.

Masuda, Takahiko and Shinobu Kitayama (2004), "PerceiverInduced Constraint and Attitude Attribution in Japan and the US: A Case for the Cultural Dependence of the Correspondence Bias," Journal of Experimental Social Psychology, 40 (3), 409-416.

Miller, Joan G. (1984), "Culture and the Development of Everyday Social Explanation," Journal of Personality and Social Psychology, 46 (5), 961-78.

Miyamoto, Yuri and Shinobu Kitayama (2002), "Cultural Variation in Correspondence Bias: The Critical Role of Attitude Diagnosticity of Socially Constrained Behavior," Journal of Personality and Social Psychology, 83 (5), 1239-48.

$\mathrm{Ng}$, Sharon and Michael J. Houston (2006), "Exemplars or Beliefs? The Impact of Self-View on the Nature and Relative Influence of Brand Associations," Journal of Consumer Research, 32 (4), 519-29.

Nielsen (2011), "January 2011: Online Video Usage Up 45\%," Nielsenwire, (February 11), (accessed July 12, 2012), [available at http://blog.nielsen.com/nielsenwire/online_mobile/january2011-online-video-usage-up-45/].

Nisbett, Richard E., Craig Caputo, Patricia Legant, and Jeanne Marecek (1973), "Behavior as Seen by the Actor and as Seen by the Observer," Journal of Personality and Social Psychology, 27 (2), 154-64.

Novak, Thomas P., Donna L. Hoffman, and Yiu-Fai Yung (2000), "Measuring the Customer Experience in Online Environments," Marketing Science, 19 (1), 22-42.

Omodei, Mary M. and Alexander J. Wearing (1990), "Need Satisfaction and Involvement in Personal Projects: Toward an Integrative Model of Subjective Well-Being," Journal of Personality and Social Psychology, 59 (4), 762-69.

Pham, Michel Tuan and Tarmar Avnet (2004), "Ideals and Oughts and the Reliance on Affect Versus Substance in Persuasion," Journal of Consumer Research, 30 (March), 503-518.

Pine, B. Joseph and James H. Gilmore (1999), The Experience Economy: Work Is Theater \& Every Business a Stage. Boston: Harvard Business School Press.

Pronin, Emily and Lee Ross (2006), "Temporal Differences in Trait Self-Ascription: When the Self Is Seen as an Other," Journal of Personality and Social Psychology, 90 (2), 197-209.

Ross, Lee (1977), "The Intuitive Psychologist and His Shortcomings: Distortions in the Attribution Process," in Advances in Experimental Social Psychology, Vol. 10, L. Berkowitz, ed. New York: Academic Press, 173-220. 
Singelis, Theodore M. (1994), “The Measurement of Independent and Interdependent Self-Construals," Personality and Social Psychology Bulletin, 20 (5), 580-91.

Spreng, Richard A., Scott B. MacKenzie, and Richard W. Olshavsky (1996), "A Reexamination Determinants of Consumer Satisfaction," Journal of Marketing, 60 (July), 15-32.

Trope, Yaacov and Nira Liberman (2003), "Temporal Construal," Psychological Review, 110 (July), 403-421. and - (2010), "Construal-Level Theory of Psychological Distance,” Psychological Review, 117 (2), 440-63.

Vallacher, Robin R. and Daniel M. Wegner (1989), "Levels of Personal Agency: Individual Variation in Action Identification,” Journal of Personality and Social Psychology, 57 (4), 660-71.
Watson, David (1982), "The Actor and the Observer: How Are Their Perceptions of Causality Divergent?" Psychological Bulletin, 92 (3), 682-700.

, Lee Anna Clark, and Auke Tellegen (1988), "Development and Validation of Brief Measures of Positive and Negative: The PANAS Scales," Journal of Personality and Social Psychology, 54 (5), 1063-1070.

Wu, Shali and Boaz Keysar (2007), "The Effect of Culture on Perspective-Taking," Psychological Science, 18 (7), 600-607.

Zhao, Min, Steve Hoeffler, and Gal Zauberman (2007), "Mental Simulation and Preference Consistency over Time: The Role of Process- Versus Outcome-Focused Thoughts," Journal of Marketing Research, 44 (August), 379-88. 